\title{
Workshop on the Application of Existing Satellite Data to the Study of Ocean Surface Energetics 19-21 November 1980, University of Wisconsin-Madison
}

\author{
Catherine Gautier ${ }^{1}$ and Dudley McConnell ${ }^{2}$
}

\section{Introduction}

The immediate purpose of the Workshop on the Application of Existing Satellite Data to the Study of Ocean Surface Energetics, held on 19, 20, and 21 November at the University of Wisconsin-Madison, was to provide a forum for discussion between data users and producers, in order to achieve improved mutual understanding of the constraints on both sides. There were, in addition, deeper and longer term goals for the workshop. These relate to gaining a better understanding of the role of the influence of the oceans in determining global climate; to developing a reliable, objective record of ocean surface characteristics; and to formulating a strategy for ocean monitoring for climate purposes.

The importance of the oceans' influence on climate has been well documented (U.S. Climate Program Plan, 1977). More recently, the Global Weather Experiment indicated there were important inter-hemisphere effects of the oceans on atmospheric circulation patterns. ${ }^{3}$ New results show a strong relationship between sea surface temperatures in the central tropical Pacific (Rasmusson and Carpenter, 1981) and upper air flow and consequent surface condition over North America (Horel and Wallace, 1981). So the importance of the oceans is well recognized.

Nevertheless, to make this understanding quantitative and useful, one needs a reliable and objective data record of the oceans. Rendering the record useful for climate imposes some severe constraints. Climate generally refers to a characterization of the weather over some period of time (from two weeks up to centuries) at some locale. Because of the central tendencies of the data, the differences in, say, the mean value of some parameter from two periods, is likely to be much smaller than the differences among individual values of the parameter (i.e., interannual signals are generally much smaller than seasonal signals). Consequently, the accuracy

\footnotetext{
${ }^{1}$ Space Science and Engineering Center, University of WisconsinMadison, Madison, Wis. 53706.

${ }^{2}$ National Climate Program Office, National Oceanographic and Atmospheric Administration, Rockville, Md. 20852.

${ }^{3}$ Private communication from Dr. Rex Fleming, Director, GARP Project Office. Now submitted for publication.

and precision of instrumental data useful for short-term purposes may be wholly inadequate for climate.

Secondly, data records become more valuable for climate as the length of the record increases. To form a useful data record over several generations of measuring instruments requires inter-comparability among the sensors or data analysis techniques to render the data compatible.

Finally, in these times of federal budget stringency, it is vital to define as carefully as possible the limits of existing data capabilities when formulating new measurement programs.

For all of these reasons, it was deemed very important to convene a workshop to review the status of applications of the existing data and to make recommendations for further, near-term efforts-both to broaden the usefulness and the utility of the data, and to define their limits in the context of the need for additional measurement systems (e.g., the National Oceanic Satellite System (NOSS), the Ice Experiment (ICEX), the Topography Experiment (TOPEX), and so forth).

The workshop was structured into six sessions: Heat Fluxes, Radiation, Topography and Currents, Wind and Wind Stress, Precipitation, and Sea Surface Temperature. All sessions consisted of one invited review presentation followed by short technical contributions. These presentations covered the first day and a half of the workshop. The next half-day was devoted to working group activities around the six topics of the sessions. The report and recommendations of each working group were presented to the entire audience for discussion and comments on the third day.

The following summary of the presentations is divided according to the sessions, with the executive summary and recommendations of each working group under the session heading. The full proceedings of this workshop can be obtained from the University of Wisconsin Press.

\section{Opening session}

Prof. Suomi opened the conference by indicating the important role existing satellite data can play in the study of the ocean surface energetics. He gave two major examples. First, the work that has been done with geostationary satellite data during the Global Weather Experiment, emphasizing the preliminary results describing the Indian monsoon circula- 
tion and the possibility of forecasting its onset. Second, the potential of the new VAS (VISSR Atmospheric Sounder), on board the recently launched GOES-4, for atmospheric temperature profile and sea surface temperature determination. VAS data analysis is in a preliminary stage, but has already shown great potential for deriving surface temperature using a double-window technique (with the "clean" $4 \mu \mathrm{m}$ channel) and taking advantage of the repetition rate of geostationary platforms. These applications are described in more detail in the technical presentations. Francis Bretherton then introduced the topic of the ocean surface energetics, and also provided the concluding remarks.

\section{Ocean Surface Energetics: Review- F. Bretherton}

F. Bretherton briefly reviewed the important role of the oceans on the climate system over various time scales, and emphasized the key elements in understanding the interface processes, i.e., the fluxes of heat at the ocean surface; the stress exerted by the wind; and, to a lesser extent, the net moisture flux (precipitation minus evaporation) on a large scale. To provide a sense of perspective, he then considered typical climatic signals. He demonstrated that a significant measurement of surface flux or poleward heat flux should be to a precision of about $10-15 \mathrm{~W} / \mathrm{m}^{2}$ on space scales of several hundreds to thousands of kilometers, and time scales of a few weeks and longer. He acknowledged that this is a very demanding condition, and unrealistic as a measurement requirement. Another view of this measurement accuracy may come from sensitivity studies made with numerical models. Sea surface temperature anomalies and the $\mathrm{CO}_{2}$ effect were discussed as examples.

Faced with such a difficult measurement accuracy, the strategy, suggested Bretherton, must be a dual one. Techniques should be developed that, on the one hand, demonstrate that they can achieve the $10-15 \mathrm{~W} / \mathrm{m}^{2}$ requirement and, on the other hand, can provide a consistent synoptic view, with measurement accuracy being less important than spatial and time coverage. This leads to the concept of the climatic index - a long time series of consistent and repeatable observations believed to have some association with some significant variable in the overall system. Candidates for such indexes were listed in a UCAR report (UCAR, 1981):

1) heat storage from the existing time series in the Pacific, extended to the North Atlantic and selectively in the southern and Indian oceans;

2) salinity and temperature fluctuation from hydrographic stations;

3) sea level from island tide gages now existing in the $\mathrm{Pa}$ cific but selectively extended elsewhere;

4) sea level differences together with either pulsed acoustic transmission or electric potential across the Florida Straits leading to the mass and heat transports by the Florida current;

5) satellite cloud drift winds throughout the tropics as an indicator of surface stress;
6) acoustic pulse travel times for a long acoustic path in the North Atlantic (e.g., Eleuthera-Bermuda) to yield information on the temperature or perhaps velocity variations at different depths;

7) temperature and velocity at depth from moorings in the Denmark Strait to monitor Norwegian Sea overflow;

8) precipitation activity derived from satellite infrared imagery and microwave radiometry;

9) serial releases of drifting buoys from selected fixed points;

10) bottom temperature and velocity from a mooring in the Hatteras Abyssal Plain to indicate the northward extent of the Antarctic bottom water;

11) electric potential measurements from an instrument towed by ships of opportunity to determine the vertically integrated heat flux.

Other implications of a measurement accuracy of \pm 15 $\mathrm{W} / \mathrm{m}^{2}$, as shown by effects in the upper layer of the ocean, which would impact the heat budget by a comparable amount, were discussed for different time scales. To close, Bretherton emphasized measurement system comparison as the central strategy, and illustrated this point using the Seasat Scatterometer. As he pointed out, only by putting much more effort than we have done in the past to crosscheck, will we make significant progress towards the twin goals of sustained indices and quantitative measurements.

\section{Session 1: Heat fluxes}

\section{a. Review-E. Kraus}

E. Kraus' presentation mainly discussed the question of whether, or how, one could assess the turbulent heat flux across the sea surface, averaged over periods of about a month's duration, and over areas of order $10^{\circ}$ square, with an accuracy of $10-15 \mathrm{~W} / \mathrm{m}^{2}$. After reviewing the expression for the total surface energy flux, Kraus discussed the various error sources, such as parameterization, sensing, and sampling errors, that are introduced when determining the surface heat fluxes. He noted that a lot of work remains to be done to account quantitatively for the influence upon the surface fluxes of secondary physical processes, such as wave formation, the role of spray, and so forth. He demonstrated the value of using the atmospheric friction velocity $U_{*}$ in parameterization schemes instead of the surface velocity $U$, particularly in the case of the scatterometer. He then showed some results of calculated turbulent heat flux, for the $D S T$ summer phase, obtained using different parameterization (Bunker, Dyer, and Kondo), in order to emphasize the large discrepancies between these nonlinear parameterization schemes.

Kraus then introduced the sampling problem, discussed the aliasing effects of weather phenomena with time scales less than $12 \mathrm{~h}$, and the small contribution of high frequency fluctuations to the total variance. Measurements taken as often as every 1.5 days seem to be necessary to provide 
monthly mean fluxes. This would suggest a minimum of about 25 well-spaced measuring stations to derive the heat flux between $25^{\circ}$ and $65^{\circ} \mathrm{N}$ in the Atlantic. A ship of opportunity program alone cannot meet such a requirement, but the diagnosis and the following-up of weather perturbations with appropriate synoptic analysis can be sufficient. This is where satellite observations can help in assessing the advection and evolution of synoptic systems, which sometimes have the effect of increasing the heat flux by more than 400 $\mathrm{W} / \mathrm{m}^{2}$.

There remains still the problem of getting the best possible surface flux distribution. The question of whether optimal statistical estimates are being obtained from the smoothing of a product (sampling method) or from prior smoothing of the factors (classical method) is not trivial, and is addressed in the next paper.

\section{b. Short contributions}

The Reynolds and Esbensen presentation dealt with quantitative testing of the differences between the classical and the sampling methods to estimate monthly-averaged air-sea transfers of heat and momentum. Using 25 years of weather ship data, they showed that the two methods agree to within a few percent in both summer and winter, and in all climatological regimes of the subtropical and mid-latitudes oceans; the sample method used was represented, within $10 \%$, by the classical method. Their belief is that the classical method of using wind roses and monthly averaged state variables to estimate wind stress and heat flux had an important advantage over the sampling method, since it is no longer necessary to obtain all the variables simultaneously. This would allow incorporation of satellite observations into the monthly average estimates.

The next presentation by $\mathrm{T}$. Liu dealt with the evaluation of parameterization models for determining air-sea exchanges of heat and momentum from satellite data. He presented a reformulation of the fluxes as a function of surface temperature and wind speed, boundary layer averaged variables, and a scaling height (possibly the boundary layer height). He then discussed, from existing results and potential capabilities of instruments, the possibility of deriving these variables from satellite data. This method, as he pointed out, should be applied first to the determination of latent heat flux, because over the ocean it is much larger than the sensible heat flux.

Prabhakara and Chang proposed another approach to estimate the latent and sensible heat fluxes from passive remote measurements. In their case, they proposed to use the measurements of the total water vapor budget in a column to determine the evaporation at the surface. This method requires the knowledge of the total precipitable water, the mean wind speed in the boundary layer, and its divergence; the evaporation then is obtained as a residual. They presented maps of mean precipitable water derived from microwave measurements, and discussed the capabilities of estimating rainfall and wind from satellites.

The last paper of this session was presented by D. Atlas with Chou and Hwang as co-authors, and covered the topic of estimating the heat transport over coastal waters from cloud street signatures. The first approach proposed was to derive the total latent heating between the shore and a downwind point where the cloud streets exist. The needed temperature of cloud top is assumed to be known from the 11 $\mu \mathrm{m}$ Advanced Very High Resolution Radiometer (AVHRR) measurements. The heat flux is obtained by multiplying the heating by the average wind speed. The second approach discussed in detail is based on the 'cloud-free path' (CFP), that is, the distance of travel of the cold air over warm water until clouds form. This method requires the use of a model of the convective boundary layer over the sea. Model results are used to compute mean surface sensible and latent heat fluxes between the shore and the first cloud, from heat and water budget equations. The variables needed are the land temperature and vapor mixing ratio, sea surface temperature, and a measurement of the CFP. The third approach is to derive the latent heating from the slope of the boundary layer top, and calculate the flux using an independent measure of the wind speed. The relationship between the average slope of the top of the boundary layer and the average fluxes is derived from the previous model.

\section{c. Working group summary and recommendations}

Despite the number of methods presented, it was recognized that no existing technique provides the heat and moisture flux information on global patterns, along with seasonal and interannual variations needed for climate studies. At present, only a simple index indication of the distribution and variability of strong heat exchange events seemed feasible, and the main recommendations of the working group were: first, encourage attempts to use existing satellite data to obtain quantitative estimates of sensible and latent heat fluxes; and second, use satellite image data to compile a cloud census record or a strong heat flux event record. This record should be developed to focus on global distributions of the strong flux events and the interannual variability of these events. Methods for relating cloud patterns and cloud types to the heat and moisture fluxes should be pursued, but the cloud census or cloud index records can be gathered at the same time, or even before the quantitative methods are developed. The philosophy is that interannual variability of the fluxes by itself is very important information. In addition, the working group felt that further theoretical and empirical studies are needed for: 1) the interpretation of scatterometer data in terms of stress or friction velocity and 2) a better discrimination between latent and sensible heat flux parameterization.

\section{Session 2: Radiation budget at the surface}

\section{a. Review-R. Ellingson}

After having mentioned briefly the importance of knowing the distribution of the net radiation budget at the ocean surface, Ellingson outlined existing inferral techniques for estimating the surface radiation budget, satellite data availability, and possible improvements to both the inferral techniques and the satellite observation systems. He first reviewed the inferral problems and discussed the limitations of the measurements, i.e., the spectral interval, the field of view, and the various geometrical (sun and satellite viewing angle) 
limitations, which lead one to rely upon radiative transfer theory to estimate the quantities necessary for the surface radiation budget studies. He then reviewed the two existing inferral techniques, pointing out their strengths and weaknesses. In one, statistical techniques are used empirically to relate surface observations to satellite radiance measurements; in the other, physical (radiative) models are used to calculate surface solar radiation budgets. These models employ climatological data to estimate the absorption and scattering characteristics of the non-cloud radiation quantities, and use satellite observations to determine cloud amount and cloud albedo. From both approaches, the available technique for estimating the mean daily solar radiation budget at the surface gives results that agree with conventional observations to within $10 \%$. In view of the uncertainties in the comparisons with conventional observations and the requirement for surface radiation budgets at time and space scales not available over the oceans from the conventional data, Ellingson concluded that the various inferral schemes can provide data useful to climate studies. Ellingson also reviewed the available satellite data, concentrating on data obtained from the NOAA polar satellites, the NIMBUS 3,6 , and 7 series, and the GOES series. He pointed out that each of the data sets had peculiarities to be considered before using the data and, particularly, the lack of certainty concerning the calibration of the visible channels on both the NOAA and GOES satellites requires caution in using the data. Finally, he mentioned some considerations concerning the future, emphasizing improvements in the modelling of the radiative transfer and the satellite data use, sensitivity tests with the models used in inferral schemes, and questions to be addressed, such as the effects of finite clouds or of cloud droplet distributions on the measured radiances. He noted that no long-wave radiation budget estimation techniques using satellite observations have yet been formulated.

\section{b. Short contribution}

Catherine Gautier presented her work on the estimation of the short-wave energy budget at the surface in the tropical Atlantic during GARP Atlantic Tropical Experiment (GATE) from geostationary satellite data. The method used is a simple radiative modelling of the atmospheric and cloud properties. Visible brightness measurements at $1 \mathrm{~km}$ resolution are used to map cloudy regions and derive cloud bulk radiative properties. Hourly surface insolation and short-wave energy budget are estimated from hourly images, and integrated in time to obtain daily values. The method provides estimates within $9 \%$ of ship measurements made during Phase III of GATE.

\section{c. Working group summary and recommendations}

The importance of the role of the radiation budget at the surface interface for the heat budget and circulation of the oceans has long been recognized. However, our knowledge of the distribution of the radiative budget is poor because of the lack of surface observations, uncertain empirical estimates, and uncertain estimates of cloudiness. However, as pointed out by Bretherton, estimation of the global heat budget requires uncertainties in the radiation budget of no larger than about $15 \mathrm{~W} / \mathrm{m}^{2}$, which is roughly $15 \%$ of the global averaged short-wave budget over the ocean, and $49 \%$ of the net long-wave budget. Therefore, in order to estimate the net radiation budget on a global scale, we must estimate the surface budget to within about $\pm 10 \%$ and $\pm 20 \%$ for the short- and long-wave budgets, respectively. However, regional scale studies may require even more stringent accuracy requirements at the daily time scale.

The recommendations of the radiation working group are as follows:

1) Verify the short-wave estimation techniques with observations over the sea for a variety of cloudiness conditions. Ellingson's review highlighted the fact that there are techniques using satellite data that can provide the surface short-wave budget at $\pm 10 \%$ accuracy at a daily time scale. However, none of these techniques has been adequately verified over the ocean. In order to accomplish the verification, it is required first to identify ship and aircraft data that might be used for this purpose. If there is an absence of such data, it will be necessary to design a strategy for obtaining such observations, perhaps from a ship of opportunity, along with observations from the GOES geostationary satellite.

2) Develop a technique for estimating the long-wave radiation budget using readily available satellite data. A glaring deficiency in the estimation of the surface radiation budget is the absence of any technique for estimating the surface long-wave radiation budget.

3) Begin research on the development of techniques to overcome the sampling problems inherent in data available from earlier polar orbiting satellites. In particular, it is mandatory that diurnal models and horizontal- and time-averaging techniques be developed with use of geostationary data, in order to make proper use of the polar orbiting observations.

4) Perform additional radiation model development and testing. In particular, it is necessary to perform sensitivity tests with the models in order to estimate the uncertainty of the models to uncertainties in the observed satellite data, and to isolate parameters that perhaps should be measured. Furthermore, many of the inferral techniques are based on very simple physical models, which may be improved by the adaptation of efficient, sophisticated radiation models.

5) Rectify deficiencies in the knowledge and understanding of many radiative properties and mechanisms. To accomplish this end, the working group recommends:

a) the study of the albedo of wind-roughened and surface-contaminated seas;

b) the study of the distribution and radiative properties of aerosols;

c) the continuation of laboratory and theoretical studies of the transfer of radiation in finite-sized cloud fields.

6) As a matter of urgency, NOAA and NASA should put forth and initiate a program for the long term continuation of the ERB monitoring program. Also, the agencies 
should initiate a program to implement new space observations that can be used to better define the surface radiation budget.

7) Treat haze concentration information of the Coastal Zone Color Scanner (CZCS) as a primary source of data of significance to estimate the short wave radiation budget. The $0.8 \mu \mathrm{m}$ channel on the CZCS is a potentially important source of information on the concentration of atmospheric haze.

\section{Session 3: Surface topography and currents}

\section{a. Review-N. Huang}

N. Huang started his presentation by noting the role of the motion of the ocean, especially in the surface layer, in determining the atmosphere and ocean interaction processes. Although results on large scale ocean circulation (inferred from shipboard hydrographic data) are voluminous, our knowledge is still limited, principally due to the lack of synoptic observations. However; the data collected have helped in understanding the mean and variation of the general circulation, and, more recently, have shown the existence of mesoscale variability. This has changed our perception of the physical processes of the ocean and increased the need for synoptic data. It is hoped that the space technologies reviewed will provide the needed synoptic coverage.

Huang briefly described satellite tracking drifts and the use of satellite imagery, but emphasized satellite-borne radar altimetry. The principle of satellite altimetry was presented together with its problems, and the focus was on the utilization of the present data. The GOES- 3 and Seasat- 1 data were discussed, and the qualities of their data compared. Due to the premature failure of Seasat, GOES-3 data, though of lesser quality, have been extensively studied. Huang presented the results of his research on the region bounded by $22-42^{\circ} \mathrm{N}$ and $60-82^{\circ} \mathrm{W}$.

Profiles of sea surface were obtained for a six-month time period, and used to estimate adjusted sea surface heights at the intersecting points of the satellite tracks. This procedure produced topographic contours then used to compute mean topographic surface height and standard deviation by two approaches: The "climate approach" and the "weather approach." Climate statistics were obtained from analysis of six-month time intervals, and weather statistics were obtained from analysis of much shorter time intervals. A threeyear mean map using the climate approach was presented in which the Gulf Stream was very well defined up to the meander region. When the current approaches the New England Sea Mountain Chain, the flow pattern was confusing, due to both geoid and bottom topography problems. The corresponding standard deviation provided an overall picture of the geostrophic variability of the circulation. The map had a general pattern consistent with Wyrtki's eddy kinetic energy map. Some discrepancies were tentatively explained by Huang. A monthly mean surface map (April 1977) was shown and compared with the three-year mean in an attempt to identify cold eddies. The depressions agreed in general with isotherm surfaces produced by NWS.

Huang closed his presentation by discussing the future development of radar altimetry. He emphasized the crucial deficiencies in geoid knowledge and frequency of coverage. $\mathrm{He}$ mentioned the use of satellite-to-satellite tracking to gain the orbit precision needed for determination of global circulation patterns. Huang also noted the use of altimeter data in an inversion method as proposed by Wunsch. Even though a lot of improvements are needed and will probably not all be undertaken in the near future, the present and future altimeter data will be very useful in determining the variations of circulation patterns.

\section{b. Short contributions}

Kao and Cheney presented their work on the Gulf Stream front and, more particularly, on a comparison of Seasat altimeter data in the Gulf Stream region were compared with height normally quantitatively determined from Seasat altimeter data in the gulf Stream region were compared with three existing models of the Gulf Stream. Specifically, the horizontal length scale that spans the sea surface rise across the front was tested, and Kao's model was found to be the most representative of the data. Lacking simultaneous subsurface hydrographic measurements, the test perhaps is not totally definitive in terms of the subsurface structure; even so, it is one of the few quantitatively meaningful applications that can be done with existing satellite altimeter data.

The next paper presented by C. Mooers dealt with the Point Sur coastal upwelling center, and its remote sensing potential and air-sea interaction question. Satellite IR imagery was used to describe several case studies of coastal upwelling, and to help clarify some notions about the generation of observed cold filamentous features of recently upwelled water. The air-sea interactions implications of the coastal oceanic surface anomalies (coastal fog, distribution of marine aerosols, sea-breeze regimes, etc.) also were discussed.

\section{c. Working group summary and recommendations}

The working group concluded that the questions which could be addressed with the present data sets are: the spacetime variation of current and eddy fields (limited area study); the space and time scale of the eddy field in the northwestern Atlantic, in order to understand the interaction between the eddy field and the mean circulation pattern; the east-west height changes across the equatorial regions; and, in combination with IR and color data, the delineation of ocean fronts, eddy and boundary current processes.

The recommendations were the following:

1) Improve the gravimetric geoid model. The geoid is the reference surface from which the oceanic dynamical signals will be measured. At present, even in the most detailed geoid, there are errors both in short wavelength features such as seamounts and trenches, and in long wavelength features, such as the general tilting. The short wavelength error will alias with the current variability. The general tilting prevents us from study- 
ing the ocean general circulation. Data from the proposed GRAVSAT experiment will help solve the general tilting problem. However, the short wavelength features must be fixed by gravity measurements.

It is believed that the present global gravimetric geoid may have an error up to $10 \mathrm{~m}$, and the present altimetric geoid may have an error up to $2 \mathrm{~m}$. A composite geoid produced from all the altimeter data and gravity data could bring the global geoid error down to around $1 \mathrm{~m}$. This composite geoid should serve as the starting point for the study of ocean current variation in the near future, and should be constructed.

2) Implement the correction in the altimeter data reduction. ${ }^{4}$ A number of error sources are known to us, but the exact ways to correct them are not established. The most serious one is the sea-state bias caused by the skewness in the electromagnetic reflectivity and ocean wave convolution. Research on this correction should be actively pursued, because sea-state change is usually on the scale of a storm that also is forcing the local current field. Furthermore, current boundaries also can produce large sea-state changes.

3) Continue the present space-time statistical study of the eddy field variability in the northwestern Atlantic Ocean. The northwestern Atlantic Ocean is the most studied region of the world ocean. GOES-3 data also are concentrated here. Continuation of the present analysis should include comparisons of the altimeter results with in situ data and numerical models, to provide an understanding of the physical and dynamical significance of the space and time scales of the eddy field.

4) Examine the discrepancy of the height difference across the Gulf Stream between altimeter measurements and the result from the dynamic method. The typical height difference across the Gulf Stream measured by the altimeter is around 1.4 to $2 \mathrm{~m}$. However, if the dynamic method based on hydrographic data is used, the height differences are around 1 to $1.2 \mathrm{~m}$. The cause of this discrepancy should be resolved. Is it due to a geostrophic motion and error in the assumption of the level of no motion, geoid inaccuracy, or altimeter errors?

5) Study the possibility of using data from near parallel paths. The next satellite carrying an altimeter may have a high inclination orbit (e.g., a NOSS-type system). The ground tracks generated by this satellite will be nearly parallel, with very few crossings. How we can best use this data set in the study of the current field of the ocean should be examined.

6) Establish an integrated oceanographic data base. Altimeter data have provided a large volume of surface measurements. In special regions, such as the northwestern Atlantic Ocean, large amounts of in situ data also are available. An effective system to indicate the existence of various types of data, and a functioning

\footnotetext{
${ }^{4}$ In private communications, officials from NASA have indicated their plan to publish an improved GOES-3 data set some time in 1981.
}

system with capability to use these data together as an integrated set to study ocean dynamic problems, is an urgent task. For example, historic hydrographic data can be used to improve the geoid, while the altimeter data could give a better synoptic space-time picture of the eddy field.

7) Use the IR and color data to study the oceanic fronts, eddies, and boundary currents. Oceanic fronts are formed by two distinct water masses coming into contact. Often, there is a strong difference in temperature and color in the different water masses. Both IR and the color scanner can be of great use in studying the movement and evolution of the oceanic fronts, especially in the coastal region and near the concentrated boundary current systems off the east and west coasts of continents. A statistical climatology of surface thermal and color patterns needs to be developed in a few diverse regimes.

8) Study the optimal mixture of satellite platform and instruments. Not all the remote sensing instruments are compatible on the same satellite platform. There are chances of signal interference, and different orbit requirements. Most of all, putting all the sensors on the same platform does not even ensure that the instruments are looking at the same spot. The following questions need to be addressed: a) Is it more advantageous to have a separated but coordinated system of satellites all launched from the same mission, as for example, a shuttle? b) What is the optimal combination?

9) Implement the multi-beam altimeter. The major shortcoming of the altimeter is the narrow swath of data collection. Therefore, for a required data density, more than one satellite is needed. A multi-beam altimeter could alleviate this need. Furthermore, an area-scanning altimeter that gives a three dimensional height measurement also should be a possibility. All these possibilities should be studied for future altimeter missions.

10) Improve other measurement methods. Satellite-tracked drifters and ships of opportunity measurement techniques should be improved, and data reporting procedures automated.

11) Study other data products from the altimeter. Other than height measurements, an altimeter also can provide data on surface wind waves, surface wind speed, precipitation, etc. These data should be analyzed from the present data set as an input to climate studies.

12) Publication of a data catalog for both GOES and SEA$S A T$ altimeter data and data products now existing.

\section{Session 4: Wind and wind stress}
a. Review-G. Philander ${ }^{5}$ and D. Halpern
G. Philander's review and Halpern's presentation discussed

\footnotetext{
${ }^{5}$ The review was not presented by G. Philander because of illness and D. Halpern kindly offered to present it at the last minute.
} 
and explained why accurate knowledge of the surface wind stress is necessary for oceanic models that predict Sea Surface Temperature (SST). In the subtropics and mid-latitudes, one-dimensional mixed layer models are remarkably successful at predicting the SST. This is not the case in the tropics. In the tropical Atlantic it has been shown, for instance, that the seasonal changes in the heat storage could not be explained locally, but are due to a basin-wide horizontal redistribution of heat. This can be illustrated also in the Pacific Ocean, where the isotherms have an east-west slope, which is the consequence of accumulation of water piled up by the trade winds. If the wind should relax, then the isotherm would become horizontal, due to the redistribution (transfer) of heat from the western to the eastern part of the basin. The trade wind relaxation occurs seasonally over the tropical Atlantic and interannually over the Pacific Ocean. Over the Indian Ocean, changes in wind conditions are abrupt and related to the onset of the monsoon. Annual (Somali current) and semiannual (eastward equatorial jet) redistributions of heat take place, which can be treated independently. Studies of the three oceans, therefore, involve measurement programs of various duration and extent, the most difficult one probably being the Pacific, because the basin is very large and the spectrum of variability is very "red."

\section{b. Short contributions}

D. Halpern continued his presentation by showing some results of correlation studies between low-level cloud motion vectors and surface wind measurements during the Global Weather Experimental (GWE). Winds produced by the National Earth Satellite Service (NESS) and the Space Science and Engineering Center (SSEC) were compared with winds from buoy data. Comparisons of prevailing wind direction provided relatively different results with southeast tradewinds observed at the buoys and more westward predominancy derived from the clouds (NESS and SSEC). Correlations between $u$ and $v$ components also were studied. Large scatter in regressions diagrams were found, as well as low correlations between pairs of values (buoys-NESS winds, buoys-SSEC winds). These results were not surprising for the region studied, because of the actual difference between surface winds and low-level winds. It was, however, unexpected that a low correlation would be found between NESS winds and SSEC winds. When averaging both data sets over five days, the correlation coefficients increased above the $50 \%$ noise level; no further improvement was obtained when the daily data were averaged further to 10 days. Some caution was advised in the study of daily variations of surface wind from variations of cloud drift winds.

D. Wylie presented the results of comparisons of methods for estimating wind stress from satellite data. Seasat scatterometer results, cloud drift-winds, and sun glitter winds were compared in two areas, the western tropical Atlantic and the eastern Pacific. The average differences were small (from 0.8 to $3 \mathrm{~m} / \mathrm{s}$ ) and the scatter varied from 0.7 to $1.9 \mathrm{~m} / \mathrm{s} \mathrm{r.m.s.}$ Separate comparisons of these wind estimates with data from moored buoys indicated a bias (high) of $0.5 \mathrm{~m} / \mathrm{s}$ for the sun glitter method, 1 to $2 \mathrm{~m} / \mathrm{s}$ for Seasat data, and 1.5 to $2.5 \mathrm{~m} / \mathrm{s}$ for the cloud measurements. A separate comparison of cloud motions and ship reports was made for the Indian Ocean, and a scatter of $2.6 \mathrm{~m} / \mathrm{s}$ was found. Some differences in the direction were noted $\left(42^{\circ}\right.$ r.m.s. $)$, with the agreement improving with the increasing wind speed. Thus, under conditions when wind stress is important (strong winds), the cloud motion data can be extremely useful.

\section{c. Working group summary and recommendations}

Wind stress information is needed on a wide range of time scales, from scales of daily atmospheric weather events to climatological scales. For studies of ocean surface waves, internal waves, and mixed-layer dynamics, stress estimates with time resolution of six hours is desirable. For studies of the major current systems of the oceans, a global wind-stress data set is needed with a temporal resolution of two months, and a spatial resolution of $1000 \mathrm{~km}$. Long records of these global stress data sets are needed to describe the interannual variability of the ocean general circulation.

Three methods for estimating surface stress seem to show some promise: 1) measurements of microwave backscattering by the ocean surface (radar scatterometer), 2) cloud motion winds measurements (GOES image data), and 3) measurements of microwave emission by the ocean surface (SMMR).

Improvements of these methodologies are, however, still needed. The scatterometer directional ambiguity should be removed and its measurements calibrated directly to stress. Studies of methods relating cloud motion data to surface winds need to be continued. Methods for identifying regions where the boundary layer differs from the statistics, and for predicting the boundary layer wind shear from satellite data should be developed. Tests of the accuracy of the wind estimates from SMMR need to be done, along with global verification by comparison with other methods, under a wide range of conditions. This method can provide only the scale of stress magnitudes; directions will have to be obtained from a separate source.

The specific recommendations of the wind stress working group are as follows:

1) Develop climatologies describing the global distributions of wind stress patterns. These climatologies should be made from the three-month SEASAT scatterometer (SASS) record and from the NOAA/NESS and University of Wisconsin-Madison cloud motion data sets. The two-year SMMR record from NIMBUS-7 also should be considered after verifications of the SMMR technique have been made.

2) Continue comparisons between all methods of estimating wind stress, scatterometer, cloud motions, and ship reports, and develop methods for combining these data.

3) Develop better relationships between wind speed and surface stress. More theoretical work on the relationship between capillary wave formation and stress is needed. Where possible, the data from past experiments (Air Mass Transformation Experiment (AMTEX), Joint Air-Sea Interaction Experiment (JASIN), Storm Transfer Response Experiment (STREX), and GATE) should be used to refine wind speed-stress relationships. 
4) Calibrate satellite scatterometer directly to the stress, and bypass atmospheric boundary layer dynamics, if possible. This area may require more data to be taken, because most of the past stress observations (direct observations of stress not inferred from wind data) were not taken with the SEASAT scatterometer data.

5) In all methods of obtaining global stress data sets, detailed documentation of the methods should be given top priority. The documentation should include descriptions of sensor biases, and the types of situations that cause changes in the sensor biases. Any information that may affect the validity of the data should be included.

\section{Session 5: Precipitation}

\section{a. Review-C. Ramage}

C. Ramage introduced his topic by noting that satellite data have been successfully used for studies having oceanographic or meteorological relevance. Long-time series from uniform instruments compensated for calibration drift are the only satisfying products for climatology. For the purpose of describing and understanding climate change as reflected in ocean rainfall, there is no alternative to satellite-based measurements. Ramage then reviewed the various techniques presently used. Nephanalyses were the first products used for deriving monthly average cloudiness distributions and to represent rainfall over the western oceans. They were not used over the eastern oceans, where extensive stratus sheets prevail.

Quickening interest in ocean rainfall, engendered by the great El Niño of 1972-73, led to the use of the frequency of "Highly Reflective Clouds" (HRC), as shown in satellite cloud mosaics, as a measure of rainfall. Over the tropical oceans, monthly HRC frequencies were found to be correlated significantly with monthly coral island rainfall. In the GATE area, HRC and radar-derived rainfall volume also showed a good relationship. Correlations also were found between monthly HRC frequencies and convergence of the mean surface winds (tropical eastern Pacific). When averaged over 12 months, meridional profiles of surface wind convergence, $200 \mathrm{mb}$ divergence, and HRC frequency are nicely in phase. In the vicinity of the north Pacific nearequatorial convergence zone, average rainfall calculated from low-level moisture convergence is close to average rainfall deduced from HRC frequency.

Another way of estimating rainfall is to use microwave data from the Electrically Scanning Microwave Radiometer (ESMR). First comparisons between areal distributions of the frequency of cold cloud tops (high IR brightness) and HRC frequency for the GATE A scale showed a good match between the two techniques under certain threshold conditions. This IR procedure could be automated readily, and could replace the human HRC procedure. In addition, since IR data are available twice daily, they might be used to improve HRC estimates when rainfall undergoes large diurnal variations. In conclusion, if one assumes that for most cli- mate studies a minimum interval of a month is adequate, the simple inexpensive methods just presented can be used to delineate ocean rainfall, and to define anomalies or trends. What is essential is that a prolonged homogeneous record can be achieved by adequate planning of operational satellites.

\section{b. Short contributions}

D. Martin discussed clouds, rainfall, and patterns of brightness. He first stated the rationale for estimating convective rainfall by means of visible or IR data. Then he reviewed some direct observations of clouds to illuminate the physical underpinnings for visible-infrared satellite rain estimation.

The main conclusions from the studies and observations analyzed were that:

1) In warm marine clouds, probability of rain is closely coupled with cloud thickness; however, dropsize distribution and height of cloud base may significantly modulate this relationship. Rain intensity bears a similar but weaker relationship to thickness.

2) To demonstrate skill in satellite estimation of rain, a technique based on infrared or visible images must either explain at least $2 / 3$ of the variance in $6 \mathrm{~h}$ synoptic rainfall, or offer comparable accuracy with fewer images, or comparable accuracy with better than synoptic resolution.

3) There is much insight to be gained from studies of cloud physics (and radiative transfer) in relation to the assumptions that underlie each of the visible-infrared satellite rain techniques. The need is less for Edisons than it is for Newtons.

Augustine and Griffith presented some evaluation of the Griffith-Woodley rain estimation technique in the tropics. This empirical diagnosis technique, in which histories, area, and minimum blackbody temperature of clouds are related to rainfall, is applied on a cloud-by-cloud basis with a method of apportionment for tropical rain. The performances of this technique were tested on FACE and GATE data, and the results were that the unadjusted satellite-derived volumes were 10 to $30 \%$ of rain estimates obtained either from radar or from rain gages. Further evaluations of the technique that were reported dealt with the relative errors introduced by degrading the spatial and temporal resolution of the data. Difficulties were found in determining the absolute error of satellite rain estimates, because of the error of the ground measuring system. A method of quantifying the absolute error of satellite rain estimates was presented: This was also a way of measuring the improvement of satellite estimates over the gage-measured rainfall.

This session was closed by K. Katsaros' presentation on Seasat Scanning Multichannel Microwave Radiometer (SMMR) atmospheric water determinations. Integrated water vapor results from Seasat were compared with estimations from radiosondes in the Gulf of Alaska and in the tropics. For integrated atmospheric liquid water and precipitation rate, which also can be derived from SMMR, virtually no surface intercomparison data exist. Comparisons between GOES visible data and liquid water content showed qualitative agreement. The location of rain cells in frontal storms in the 
eastern Pacific is very reminiscent of some description of cyclonic rain bands.

\section{c. Working group summary and recommendations}

Except as it influences ocean dynamics through salinity, precipitation makes no direct contribution to the energy balance at the surface. However, it is indispensible in evaluating convective parameterization schemes in global circulation models. Precipitation (hereafter, rainfall) also is an important element of phenomenological studies, for example, El Niño (where the relation between anomalies of sea surface temperature and rainfall is of interest). It is of critical importance as climatic index. A distant prospect is use of rainfall, at least on global or hemispheric scales, to infer evaporation.

Except in favored sites and for periods of a year or longer, the achievement of $\pm 10 \%$ absolute accuracy in the measurement of rainfall is not possible by any means. We may achieve $\pm 10 \%$ relative accuracy in monthly rainfall by applying a single technique to a uniform record of satellite data; this remains to be demonstrated.

Of the dozen or more methods which recently have been used to extract information on rainfall from satellite data, only three have shown promise for ocean climatology. The life history technique, which depends on infrared picture sequences from geostationary satellites, has been configured by Griffith and Woodley to run automatically on a digital computer. However, the need for high-time resolution and accurate navigation (image registration) of data, as well as large computer demands, presently limit the possibilities for applying this method on climatological scales. In the tropics, the HRC method of Kilonsky and Ramage, which depends only upon once-daily mosaics of visible pictures produced routinely by NESS, offers, at much less cost, an accuracy in monthly estimates comparable to that of the Griffith and Woodley technique. Monthly rainfall on a $1^{\circ} \times 1^{\circ}$ grid is now available for all the tropical oceans for all but a few months since 1971. The HRC technique applies to the equatorial belt between about $25^{\circ}$ north and south. Poleward of these latitudes, the most likely source of useful rain information is passive microwave observations from instruments such as ESMR- 5 because, in temperate and polar latitudes, the field of view, sampling, and rain model problems that have frustrated attempts to estimate tropical convective rain are much less serious. Thresholding techniques, like that described by Lovejoy and Austin, which depend upon infrared as well as visible imagery, hold promise for rain estimation in higher latitudes; indexing techniques such as the one proposed by Barrett may also be useful.

Easily the best existing record of data is the series of ESSA/ NOAA Mercator mosaics, which dates to 1971. A second record of special interest from the operational low-orbiting polar satellites is the series of digital infrared data, which dates to 1974, except for short periods. Digital archives of geostationary satellites begin in 1977. Microwave records date to the launch of NIMBUS 5 in 1972. A record of much potential value is USAF Global Cloud Climatology-ETAC.

Recommendations of the Precipitation Working Group are as follows:

1) Identify or, if necessary, create a ground truth system over the ocean. This system might include: a) better ship rain gages; b) microwave communication receivers on ships; c) coastal radars with differential polarization reflectivity; d) gages on marine platforms.

2) Continue HRC mapping in the tropics. Test the possibility of using infrared "cold cloud" frequencies in lieu of HRC to eventually replace HRC and automate the technique.

3) Evaluate measurements of rainfall in polar latitudes by means of passive microwave observations. This might be done in two stages: a) evaluate a few months of SMMR data against coastal radars and $b$ ) if this test is successful, reanalyze ephemeris corrected ESMR-5 data over the polar ocean. Also, insure that information on rainfall is preserved in the processing of data from the Large Antenna Multichannel Microwave Radiometer of the National Oceanic Satellite System (NOSS).

4) Continuously check satellite estimates against ground truth observations.

5) Apply knowledge of the precipitation process and models of radiative transfer in clouds to understand better the physical basis of present methods. Conduct tests to determine sensitivity of satellite estimates to sampling in time and space and to elements of technique design.

6) Derive rainfall as an additional element of the International Cloud Climatology Project.

7) Encourage the development and testing of new techniques such as: a) inferring rainfall from measurements, at microwavelengths, of the change in surface emissivity due to roughening by raindrops and reduction in salinity; b) inferring rainfall from measurements of attenuation in communication and geostationary satellites in centimeter wavelengths; c) inferring rainfall from measurements of attenuation in transmissions of radar altimeters on low orbiting satellites; $d$ ) inferring rainfall from measurements of acoustic noise produced as raindrops impact the surface of the sea.

\section{Session 6: Sea surface temperature}

\section{a. Review paper-S. Larson}

S. Larson first reviewed the potential role of SST on the climate system, mentioning the major cooling in the last great ice advance and the more immediate influence of SST anomalies on weather variability. He discussed the El Niño event and its presently proposed mechanism, Bjerknes' theory on the link between tropical and mid-latitude changes in atmospheric circulation and the Walker and Hadley circulations. Modelling of the impact of SST anomalies on the atmosphere, and some recent model results, were presented. Then Larson asked the question: Are the satellites capable of detecting SST anomalies of this magnitude $\left(3^{\circ} \mathrm{C}\right)$ ? Before attempting to answer, he discussed the problems of the effects of the SST variability (in time and space) on the estimation of the satellite-derived SST accuracy. Variability occurs at all scales, which influences comparison with ship measurements. Perhaps the most fundamental legitimate causes for the discrepancy between satellite and bulk SST is that of the 
"cool skin" of the sea. Comparisons with ship data also must take into account injection temperature bias; weather ship data show better agreement with buoy data than do merchant ship data. Coming back to his initial question, he addressed the issues of the water vapor and cloud contamination in the satellite-derived SST. The problems of changing satellites, sensors and software also were mentioned in the discussion. In summary, Larson made the following points:

1) SST anomalies, typically $3^{\circ} \mathrm{C}$, can generate anomalous atmospheric conditions both locally and at a distance, and at least a season after their occurrence.

2) In situ measurements, including “injection” SSTs from merchant vessels, are accurate enough to resolve SST anomalies $>1{ }^{\circ} \mathrm{C}$.

3) Only in limited areas and under infrequently occurring conditions can differences greater than a few tenths of a degree between in situ and satellite SSTs be explained as variations in the true SST field.

4) Satellite-derived SSTs, because of atmospheric attenuation, cloud contamination, and evolving computer methods, have a space- and time-dependent bias relative to in situ measurements.

\section{b. Short contributions}

William Smith discussed the advantages of geostationary satellite infrared observations of the SST. He discussed three possible approaches to achieve the necessary correction for water vapor absorption: 1) the extrapolation of simultaneous observations of an area from two (or more) Geostationary Earth Orbiting (GEO) satellites to zero air mass; 2 ) the use of coincident multispectral window data from polar orbiting satellites to provide a water vapor correction field for the geostationary window observations; and 3) the use of multispectral window radiance observations from the new VAS on GOES-4. He discussed the advantages and disadvantages of each method, and also recommended blending IR data with microwave data for an improved estimate of SST.

Paul McClain presented some results of global tests of a two-window method for satellite-derived SST. This method uses radiance measurements at 3.8 and $11 \mu \mathrm{m}$. Under a limited test, this technique yielded SSTs with an r.m.s. difference of $0.9^{\circ} \mathrm{C}$ compared to buoy measurements. Then a twomonth global test was run and results were presented which showed significant and consistent improvements from the present GOSSTCOMP product.

Thomas Chester discussed SST measurements from the SMMR. The SMMR resolution for SST is $150 \times 150 \mathrm{~km}$, which limits the comparisons with surface observations. Results were presented for four major geographical areas; the overall r.m.s. difference with buoys and ship measurements was $0.9^{\circ} \mathrm{C}$. Finally, Chester outlined the limitations of the present microwave measurements for SST estimates.

\section{c. Working group summary and recommendations}

SST plays an important role in the interactions between the oceans and the atmosphere, and is a key parameter for the determination of the surface energy balance. Small surface temperature changes in the tropical regions might, for example, influence the weather in mid-latitudes or modify seasonal atmospheric phenomena such as the monsoon. An accurate knowledge of the SST is therefore of critical importance for a large variety of climate studies over a wide range of space and time. For global climate monitoring, SST monthly averages over $250 \mathrm{~km}$ are required; for climate modelling, a spatial resolution of $100 \mathrm{~km}$ and averages over a week are needed. In the case of regional modelling, much better resolution must be obtained with a minimum of $25 \mathrm{~km}$ and averages over five days. Accuracies of $1^{\circ} \mathrm{C}$ absolute and 0.25 to $0.5^{\circ} \mathrm{C}$ relative are crucial to all these applications.

Because of the importance of SST in climate modelling and monitoring, a large effort has been expended on the measurements from satellite. SSTs are routinely produced over space and time scales specified above, but with accuracies far from those required. In view of this situation, the working group has addressed specifically the problem of improving the present operational data analysis system and that of implementing new approaches. In addition, since 1) an accurate knowledge of SST is crucial to a large class of climate studies and 2) SST data are produced operationally by NESS, the working group suggests that special consideration be given to the specific requirements for climate in the operational processing of satellite data.

Recommendations of the SST working group are as follows:

\section{A) Improvement to contemporary data analysis}

1) Develop and implement techniques to improve the cloud filtering. One of the problems in determining SST from infrared measurements comes from cloud contamination. Two methodologies are suggested: a) during daylight use visible observations; b) during the night use the difference between the $3.8 \mu \mathrm{m}$ and $11 \mu \mathrm{m}$ brightness temperature data.

2) Improve water vapor corrections, particularly in the case of GOES data. Two methodologies are suggested: a) use simultaneous measurements from two geostationary satellites; b) use simultaneous measurements from a geostationary and a polar orbiting satellite.

3) Improve calibration monitoring. Most of the IR data used for sea surface determination come from sensors imperfectly calibrated.

4) Document software changes. It is important for climatological use of the data set (here one needs a consistent data set) that any change in the software be documented and disseminated to the scientists.

5) Prepare for operational use of VAS. The VAS on board the most recently launched geostationary satellite (GOES-4) combines the several IR window channel measurements with the repetition rate of a geostationary satellite, and therefore provides a promising data source for SST estimates in the near future.

B) New approaches

1) Combine infrared and microwave approaches. This type of research is in progress, but requires some optimization of the procedure. It is particularly 
important for global monitoring for which only one approach is not sufficient to obtain the required coverage.

2) Improve archival and data retrieval procedures. Due to the need for long time series, it is important that valuable data sets such as TIROS-N and NOAA-5 be archived.

3) Improve low-cost ship and buoy data collections using satellite communications. The ASDAR concept and the NWS "Seas" prototype have been tested, but an optimization of the approach is still needed.

4) Re-program TIROS-N processor to select maximum and minimum brightness temperatures rather than the average over $4 \mathrm{~km}$ area. The present programing averaging is not adequate for optimized SST retrieval.

5) Use man-machine approach in particular for regional studies where the amount of data to handle is manageable.

In addition and most important: Recognizing that the resolution, frequency and accuracy requirements of satellite data for climate conflict with the requirements for daily analysis of meteorological and oceanographic variables, the working group recommends that operational satellite data processing centers institute new procedures leading to a separate set of observations optimized to meet the requirements for climate application.

\section{Conclusion}

It is clear from the presentations and working group recommendations that existing satellite data provide the basis for very interesting and promising research. However, time and again there arise problems in the basic data sets themselves and in the interpretation of the data. The data problems must be addressed by the data trustees-NASA and NOAA. As trustees, they, along with the scientific community, must place greater emphasis on furnishing consistent, comprehensive, documented data sets. The publication of the ozone data set from NIMBUS might serve as an example for a comprehensive corrected GOES-3 data compilation. NOAA should pursue themselves and encourage extramural development and testing of techniques to improve the usefulness of data from the operational satellite. Already, with judi- cious processing, uneven quality data sets have provided information highly relevant to climate research (e.g., HRCs for precipitation indexes over the tropical Pacific, cloud winds, and a polar orbiting satellite cloud climatology). Furnishing high quality data for climate studies must be a requirement. As was suggested by Bretherton during the closing session, the key to better understanding of data capabilities lies in more systematic comparison of methods of observation and information extracted from the data. However, systematic and consistent comparisons require that the satellite data be available in their most useful and reliable forms.

Secondly, several of the Working Groups (viz: Radiation, Precipitation, SST) referred to the need for a cloud climatology archive. The formation of such an archive is a major initiative of the National Climate Program and of the World Climate Research Program. The initiative should be pursued vigorously. Furthermore, the cloud climatology archive represents an approach to preserving enough of the intermediate and basic data products so that as data analysis and interpretation techniques improve, the improved techniques may be applied to a long record of data.

Finally, it must be concluded at this time that if satellite data records are to be of proven value for climate, the data must be taken in concert with more extensive in situ data. The satellite sensor development and data interpretation research must go forward hand-in-hand with surface and sub-surface measurement technique developments. These are not competing but complementary data sources, as was shown during the First GARP Global Experiment (FGGE).

\section{References}

Horel, J. D., and J. M. Wallace, 1981: Planetary-scale atmospheric phenomena associated with the southern oscillation. Mon. Wea. Rev., 109, 813-829.

Rasmusson, E. M., and T. H. Carpenter, 1981: Variations in tropical sea surface temperature and surface wind field associated with the southern oscillation/El Niño. Submitted to Monthly Weather Review.

UCAR, 1981: Technical studies related to the development of a system for ocean climate monitoring, Vol. I. University Corporation for Atmospheric Research, Boulder, Colo.

United States Climate Program Plan, 1977: Federal Coordinating Council for Science, Engineering and Technology. Washington D.C. 